\begin{tabular}{llc}
\hline \multicolumn{2}{c}{ Table 1 } \\
The $\tau_{3} \begin{array}{c}\text { lifetime of positrons in teflon } \\
\text { at } 1.25{ }^{\circ} \text { and } 4.2^{\circ} \mathrm{K} .\end{array}$ \\
\hline Run & Temp. & $\tau_{3}$ \\
& $\left.\rho_{\mathrm{K}}\right)$ & (ns) \\
\hline 1 & 4.2 & $1.83 \pm 0.11$ \\
1 & 1.25 & $2.17 \pm 0.13$ \\
2 & 4.2 & $1.83 \pm 0.09$ \\
2 & 1.25 & $2.17 \pm 0.12$ \\
3 & 4.2 & $1.79 \pm 0.05$ \\
3 & 1.25 & $2.19 \pm 0.07$ \\
3 & 1.25 & $2.16 \pm 0.10$ \\
3 & 4.2 & $1.84 \pm 0.06$ \\
\end{tabular}

For example, in teflon at room temperature there is a slight unwinding of the chain of CF2 groups from a twist of $180^{\circ}$ per $13 \mathrm{CF}_{2}$ groups to a $180^{\circ}$ twist per $15 \mathrm{CF}_{2}$ groups. A separation of the chain axes also occurs [4]. This transition results in a significant change of the positron lifetime. The specific heat of teflon has been measured at low temperatures. It has been found that in the temperature region of $2.5^{\circ} \mathrm{K}$ the slope of $c / T$ versus $T^{2}$ curve changes. In the temperature interval of $3.3^{\circ} \mathrm{K}$ to $4.3^{\circ} \mathrm{K}$, the curve is a straight line with a slope of $7.6 \times 10^{-6} \mathrm{cal} / \mathrm{g}^{0} \mathrm{~K}^{4}$, and in the temperature interval of $1.2^{\circ}$ to $2.0^{\circ} \mathrm{K}$, the curve is a straight line with a slope of $1.2 \times 10^{-5} \mathrm{cal} / \mathrm{g}^{{ }^{0}} \mathrm{~K}^{4}[5]$.

We suggest that the anomalous rise in the positrons' lifetime at $1.25^{\circ} \mathrm{K}$ may be due to a very low

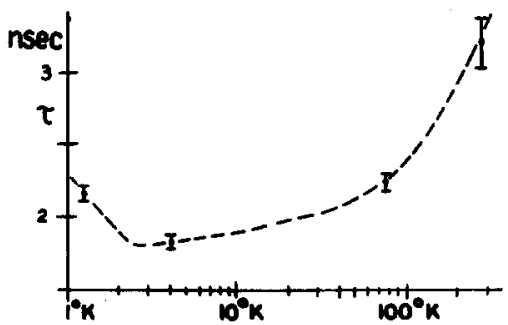

Fig. 1. Temperature dependence of the $\tau_{3}$ lifetime of positrong annihllating in teflon.

temperature transition in teflon, for positron annihilation lifetime studies at room temperature have shown that the lifetime depends upon the structure of teflon, and specific heat measurements indicate a change in slope of a $c / T$ versus $T^{2}$ curve at $2.5^{\circ} \mathrm{K}$.

Experiments are being planned to measure both the intermediate and long lifetime of positrons as a function of the crystallinity and temperature of the teflon.

\section{References}

1. P.C.Simms, Rev. Sci. Instr. 32 (1961) 394.

2. A.W. Sunyar, Bull. Am. Phys.Soc. 9 (1964) 394.

3. G. Fabri, E.Germagnoli and G. Randone, Phys, Rev, 130 (1963) 204.

4. C.A.Speratl and H.W. Starkweather Jr. , Advances in Polymer Science 4 (1961) 465.

5. R.J.Noer, thesis, Amherst College (1959).

\title{
OPTICAL IMAGE SYNTHESIS (COMPLEX AMPLITUDE ADDITION AND SUBTRACTION) BY HOLLOGRAPHIC FOURIER TRANSFORMATION
}

\author{
D. GABOR \\ Imperial College of Science and Technology, London \\ and \\ G. W. STROKE, R. RESTRICK, A. FUNKHOUSER and D. BRUMM \\ The University of Michigan, Ann Arbor, Michigan
}

Received 10 July 1965

The principles of wavefront-reconstruction imaging, first suggested and demonstrated by one of us in 1948 [1-3], have recently been successfully extended to the solution of spatial filtering problems in coherent light [4-6], along the principles first suggested by Maréchal and Croce in $1953[7,8]$. A 'true' optical filtering process may require 'complex' addition of images (or of their diffraction patterns)(rather than filtering with masks, or 'complex' multiplication of dif- 


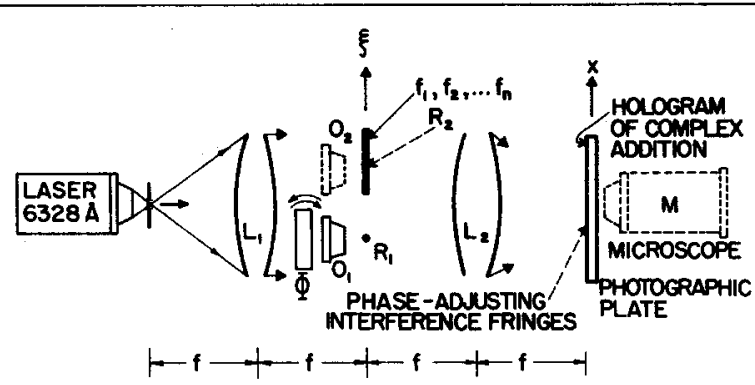

Fig. 1. Arrangement used for image synthesis by holographic Fourier transformation.

fraction patterns, used in correlation filtering heretofore). Complex 'image' addition (and, or subtraction) may be called an 'optical imagesynthesis' process. The method described here is based on the principles of Fourier-transform holography $[6,9-11]$.

For analysis, consider a one-dimensional model. By adding, in $n$ successive exposures $I_{n}$, the diffraction patterns of the image-transmittance functions $f_{n}$ to the diffraction pattern of a reference delta function $A \delta(0)$, the hologram exposure is $I=\Sigma t_{n} l_{n}$, where $I_{n}=F_{n} F_{n}^{*}+A F_{n} \exp [-2 \pi \mathrm{i} a x]+$ $+A F_{n}^{*} \exp [2 \pi \mathrm{i} a x]$, with $a=$ 'off-set' between $f_{n}$ and the point-reference $R_{1}$ (fig. 1), and $F_{n}=$ Fourier-transform of $f_{n}$. By the now usual expansion $[6,9,12]$, the hologram-transmittance in the upper first side-band is

$$
\begin{aligned}
H(x)_{1} \approx\left(t_{n}\right)^{-\frac{1}{2} \gamma_{A}-\gamma}\left(\frac{-\frac{1}{2} \gamma}{n A} \sum_{n} F_{n}+\right. & \\
& \left.+\frac{\frac{1}{2} \gamma\left(\frac{1}{2} \gamma+1\right)}{n^{2} A^{3}}\left(\sum_{n} F_{n} F_{n}^{*}\right) \sum_{n} F_{n}+\ldots\right),
\end{aligned}
$$

which gives the desired 'image synthesis', in the focal plane of a lens, by Fourier transformation of the first term (the "signal" $=S)$. ( $\gamma=$ gamma of the film.) The individual exposure times, $t_{n}=t$ here, are adjusted to avoid film saturation). Success of the complex addition $\Sigma_{n} F_{n}$ depends (as verified by experiment) on the high "signal"/ "noise" ratio, characteristic of the method. With the second term in eq. (1) taken as "noise" $=N$, one finds $S / N=n A^{2} /\left[\left(\frac{1}{2} \gamma+1\right) \Sigma_{n} F_{n} F_{n}^{*}\right]$.

The arrangement used in our experiments is sketched in fig. 1, and an example of image synthesis, obtained by 'subtracting' the complex image-transmittance function $f_{2}$ from the complex image-transmittance function $f_{1}$ (see fig. 2a) appears in fig. $2 \mathrm{~b}$. An essential element is the 'phase plate' $\Phi$, used (in this case) to obtain the desired 'subtraction' by adding $f_{2}$ with a phase shift of $\pi\left(180^{\circ}\right)$. (The $\Phi$ adjustment is straightforward, by means of interference fringes formed

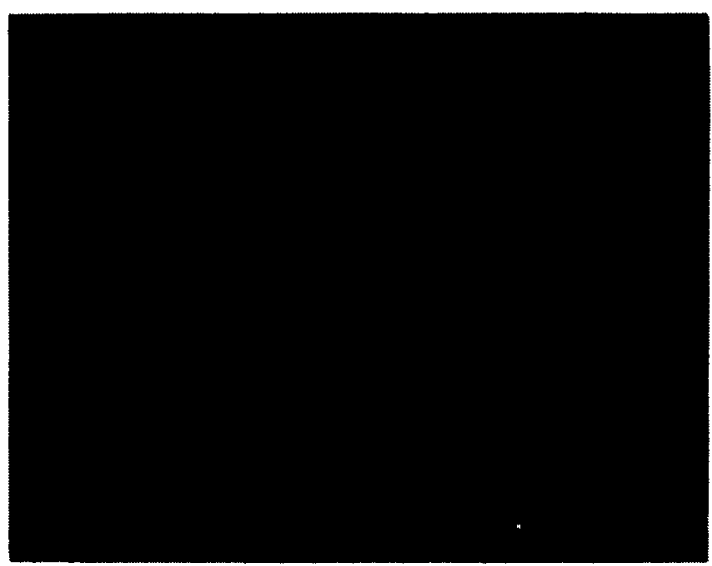

Fig. 2a. Photograph of functions $f_{1}=$ "Fourier subtraction" and $f_{2}=$ ". . urier, ... raction" of which the complex diffraction patterns were subtractively added according to fig. 1 . The hologram was recorded by adding, successively, the coherent background to the Fourier trangforms of $f_{1}$ and of $f_{2} \exp (-\mathrm{i} \pi)$.

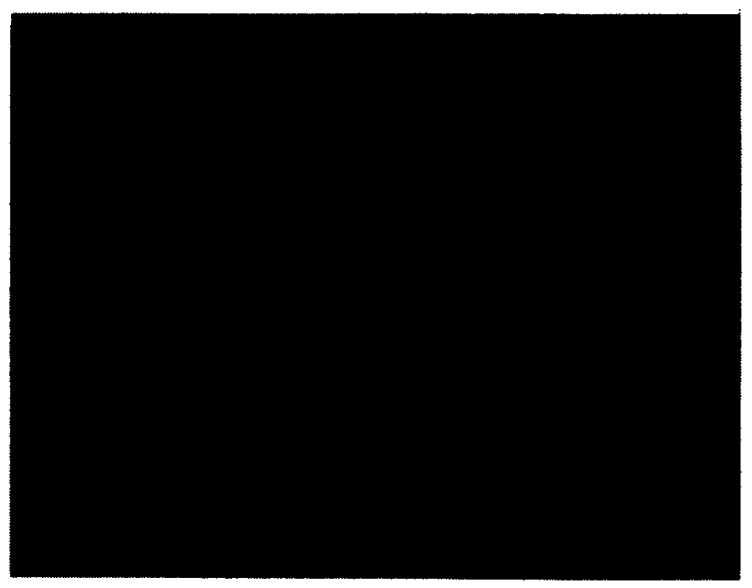

Fig. 2b. Fourier-transform reconstruction of the 'synthesized image' (here the subtractive addition), obtained in the focal plane of a lens by projecting a plane, monochromatic (6328A) wave through the hologram recorded according to fig. 1 and fig. $2 a$. The unattenuated d.c. term, together with the two side-band images are shown. The arrow points to the remaining $f_{1}$-portion, resulting from the use of a $\Phi$ shift slightly different from $\pi$ here, demonstrating the principle.

in the $x$-plane, with a temporary, auxiliary pointreference $R_{2} \cdot O_{1}, O_{2}$ are microscope objectives). The entire interferometric hologram-recording process was carried out in $6328 \AA$ laser light on Polaroid $\mathbf{P} / \mathrm{N}$ film, without any liquid gates (which may be important in some cases).

This work was supported, in part, by a kind assistance from the Office of Naval Research. 


\section{References}

1. D. Gabor, Nature 161 (1948) 777.

2. D.Gabor, Proc.Roy.Soc. (London) A197 (1949) 454.

3. D. Gabor, Proc.Roy. Soc. (London) B64 (1951) 449.

4. L. J.Cutrona, E.N.Leith, C.J. Palermo and L.J. Porcello, IRE Trans. Inf. Theory, June 1960, p.386.

5. A. Vander Lugt, IEEE Trans. Inf. Theory, IT-10, April 1964, p. 139.

6. G.W.Stroke, An introduction to optics of coherent and non-coherent electromagnetic radiations, University of Michigan, 2nd ed.1965, p.76.
7. A. Marechal and P. Croce, Comptes Rend. 237 (1953) 607

8. P. Croce, Thesis, Paris (1956).

9. G. W. Stroke and D. G. Falconer, Physics Letters 13 (1964) 306.

10. G.W. Stroke, Appl. Physics Letters 6 (1965) 201.

11. G.W.Stroke, D. Brumm and A. Funkhouser, J.Opt. Soc.Am. 55, 10 (1965).

12. G.W. Stroke, Optical information processing, eds. J. T. Tippett, I.C. Clapp, D. Berkowitz and C.J. Koester (M. I. T. Press, 1964) in print.

\title{
ON THE INTEGRAL EQUATIONS FOR FABRY-PEROT INTERFEROMETER MODES *
}

\author{
W. STREIFER \\ Electrical Engineering Department, University of Rochester, \\ Rochester, New York
}

Received 22 July 1965

There has been some disagreement lately concerning two integral equations whose solutions are Fabry-Perot interferometer modes. Lotsch [1] has stated that his equation, which describes the modes by the midplane patterns, and the earlier one formulated by Fox and Li [2] for the field patterns on the mirror (actually in the aperture of the equivalent transmission system) are essentially different. The latter authors [3] have shown mathematically that the two equations yield the same results in the sense that the field pattern on the mirror which satisfies their equation produces the pattern on the midplane which satisfies Lotsch's equation and conversely. Nevertheless in a recent letter Lotsch sets forth five arguments which he states indicate that basic dissimilarities exist in the formulations. Since nothing in his communication [4] actually demonstrates an error in Fox and Li's equivalence proof the question should be closed, but to insure that there is no doubt of the equivalence we propose, in what follows, to examine each of Lotsch's points and show them to be either invalid or irrelevant.

1) In this first item Lotsch evaluates his kernel $K\left(x_{2}, x_{0}\right)$,

$$
K\left(x_{2}, x_{0}\right)=2 F \exp \left\{i \pi F\left(x_{2}-x_{0}\right)^{2}\right\} \int_{-1}^{+1} \exp \left\{14 \pi F\left[x_{1}-\frac{1}{2}\left(x_{2}+x_{0}\right)\right]^{2}\right\} \mathrm{d} x_{1},
$$

asymptotically for large values of the Fresnel number $F$, to obtain

$$
K\left(x_{2}, x_{0}\right) \sim \exp \left\{\mathrm{im} F\left(x_{2}-x_{0}\right)^{2}\right\}\left\{\sqrt{ } F+(D / 4 \pi) \exp \left(-1 \frac{3}{2} \pi\right)\right\},
$$

where

$$
\begin{gathered}
D=\frac{E^{+}}{1+t}+\frac{E^{-}}{1-t}+\sum_{n=1}^{\infty} \frac{(2 n-1) !}{(8 \pi F)^{n}} \exp \left(-\frac{1}{2} \mathrm{inn}\right)\left[\frac{E^{+}}{(1+t)^{2 n+1}}+\frac{E^{-}}{(1-t)^{2 n+1}}\right], \\
E^{+}=\exp \left\{14 \pi F(1+t)^{2}\right\}, \quad E^{-}=\exp \left\{-14 \pi F(1-t)^{2}\right\},
\end{gathered}
$$

and

$$
t=\frac{1}{2}\left(x_{0}+x_{2}\right)
$$

* Editor's note: This diøcussion is now closed. 